\title{
Aplicación de las espectroscopias IR/ATR y Raman al estudio de la superficie de vidrios sometidos a molturación
}

\author{
S. PÉreZ-Villar, A. TAMAYO, M. A. MAZO, F. RUBio, J. RUBiO \\ Instituto de Cerámica y Vidrio. CSIC. Campus Cantoblanco. \\ C/ Kelsen, 5. 28049 Madrid.
}

\begin{abstract}
El proceso de molienda de sílice vítrea y un vidrio convencional se ha estudiado mediante espectroscopias IR/ATR y Raman. A partir de los espectros obtenidos para diferentes tamaños de partícula se calcularon tanto las posiciones como las áreas de las bandas que aparecían en cada espectro. Utilizando los espectros Raman se ha hecho uso del denominado Índice de Polimerización (IP) el cual es indicativo del grado de despolimerización producido en la superficie de las partículas tras el proceso de molienda. Por otro lado, mediante los espectros IR/ATR y analizando las posiciones de las bandas de tensión simétrica y asimétrica de los enlaces Si-O-Si se ha podido seguir el citado proceso de molturación. Se ha observado, mediante ambas técnicas, como la superficie de las partículas se despolimeriza al disminuir el tamaño de partícula. Esta despolimerización es debida a la rotura de los enlaces Si-O-Si.
\end{abstract}

Palabras clave: Raman, IR/ATR, molienda, despolimerización, silice, vidrio.

Application of the Raman and IR/ATR spectroscopies to the study of the glasses upon grinding.

The grinding process of vítreous silica and a conventional glass has been studied by means of Raman and IR/ATR spectroscopies. By using the obtained spectra, the positions and areas of the different bands have been calculated. The Raman spectra have been analysed using the Polymerisation Index (PI) that indicates the depolymerization degree achieved on the particle surface after grinding. On the other hand, the IR/ATR spectra have been used to follow the positions of the symmetric and antisymmetric Si-O-Si bands. It has been observed that both techniques give similar results. These indicate that the depolymerisation degree increases with the grinding process. This depolymerisation is due to the broke of $\mathrm{Si}-\mathrm{O}-\mathrm{Si}$ bonds.

Keywords: Raman, IR/ATR, grinding, depolymerisation, silica, glass..

\section{INTRODUCCIÓN}

Es bien conocido que los procesos de molturación de materiales cerámicos y vítreos dan lugar a que dichos materiales posean superficies muy diferentes, no solo en composición química respecto a la masa del material, sino también entre las distintas partículas según sea el alcance del grado de molienda $(1,2,3)$. El conocer como es la composición o la estructura de cualquier material es de importancia fundamental tanto para muchos procesos tecnológicos como para la salud de los trabajadores que están en contacto con el material. Así por ejemplo, dada la importancia tecnológica del cuarzo en muchos procesos industriales (cerámicas, vidrio, materiales de construcción, fundiciones, silicio metálico, aleaciones férricas, etc.,) y el papel que puede jugar este material en la formación de lesiones pulmonares (silicosis) explican el por qué el estudio de su comportamiento durante los procesos de molienda haya sido objeto de un gran número de publicaciones (4). Por otro lado, en la industria de las fritas para pavimentos y revestimientos cerámicos, los procesos de molienda de dichas fritas implican que éstas puedan ser utilizadas en determinadas aplicaciones o bien para producir efectos especiales (5). Algo similar puede decirse para la industria de los pigmentos inorgánicos utilizables en multitud de aplicaciones (azulejos, resinas y plásticos, pinturas, etc.) o en las propiedades anticorrosivas, en donde la molienda tiene un papel fundamental en cuanto al comportamiento final del material (6).

El estudio de la superficie de materiales sometidos a procesos de molienda puede llevarse a cabo mediante diferentes técnicas experimentales tales como XPS, IR, DRX, $\mathrm{RMN}-\mathrm{Si}^{29}$, adsorción de nitrógeno, cromatografía inversa de gases (IGC), etc. En todos los casos se ha observado como cambia la superficie de los materiales en función del grado de molienda, si bien dependiendo de la técnica empleada la detección de tales cambios puede ser mayor. Así, por ejemplo, en los procesos de molienda de cuarzo se ha observado como son necesarias al menos 10 horas de molienda para que se produzcan distorsiones reticulares determinadas mediante DRX, sin embargo con solamente 5 horas ya se aprecian cambios en el desorden a corto alcance determinado mediante IR, o incluso amorfizaciones superficiales determinadas 
mediante el análisis de la superficie específica a partir de medidas de adsorción de nitrógeno (1). En este mismo sentido la técnica IGC ha mostrado como la molienda de alúmina da lugar a procesos de modificación superficial desde los primeros momentos de molienda, siendo esta modificación la causante de la formación de centros de alta energía (2). Estos centros superficiales energéticos son posiblemente hidoxilos que están formando una capa superficial amorfa e hidratada. Esta misma técnica de IGC ha servido para analizar como cambia la superficie de la mica cuando es molida en diferentes medios (agua, alcohol, tolueno) y diferentes tiempos (7). Se ha observado como la energía superficial aumenta con el tiempo de molienda si se utiliza agua o alcohol en el medio, mientras que si se utiliza tolueno no ocurre tal aumento. La variación de la energía superficial de la mica molida se ha correlacionado con el aumento de superficie específica ocurrida tras la molienda así como con la capacidad de intercambio catiónico de la misma (7).

De acuerdo con todo lo expuesto, se puede concluir que la superficie de materiales inorgánicos sometidos a procesos de molienda sufre variaciones superficiales causadas por la rotura de las partículas durante dicho proceso. En este sentido, en el presente se ha estudiado el proceso de molienda de un vidrio común mediante las espectroscopias IR (ATR) y Raman. En este estudio se han analizado las diferentes bandas que aparecen en los espectros y su evolución durante la molienda.

\section{EXPERIMENTAL}

El vidrio utilizado en este trabajo fue obtenido por fusión en horno de gas a una temperatura próxima a $1500{ }^{\circ} \mathrm{C}$ durante 6 horas. La composición del vidrio es la siguiente: $\mathrm{SiO}_{2}(70.5 \%)$, $\mathrm{Al}_{2} \mathrm{O}_{3}(2 \%), \mathrm{CaO}(7.5 \%), \mathrm{MgO}(4 \%), \mathrm{Na}_{2} \mathrm{O}(16 \%)$. Pasado el tiempo de fusión, la mezcla fundida se coló sobre molde de latón, se recoció en horno eléctrico a $600{ }^{\circ} \mathrm{C}$ y se guardó en desecador hasta su utilización. Por otro lado, la sílice vítrea utilizada de elevada pureza y con un contenido en hidroxilos inferior a 30 ppm, fue proporcionada por el Departamento de Ingeniería de Materiales de la Universidad de Trento (Italia). También dicha sílice estuvo guardada en desecador hasta su molienda.

El proceso de molienda, para ambos materiales, fue llevado a cabo en mortero de ágata y en molino de atrición. Utilizando el mortero de ágata manual se trituraron los dos materiales hasta obtener tamaños de partícula comprendidos entre 300-200 $\mu$ m y 200-100 $\mu \mathrm{m}$, mientras que el mortero automático se utilizó para tamaños comprendidos entre 100-50 $\mu \mathrm{m}$ y 50-25 $\mu \mathrm{m}$. Por otro lado, el molino de atrición se utilizó para obtener tamaños de partícula inferiores a $25 \mu \mathrm{m}$. Los tamaños medios de estas distribuciones fueron para el caso de la sílice vítrea de $255,132.5,72.3,35.6$ y $8.6 \mu \mathrm{m}$, y para el vidrio de 241.8 , $126.3,67.8,31.5$ y $8.6 \mu \mathrm{m}$. Las partículas molidas se tamizaron y se guardaron en desecador hasta realizar las medidas espectroscópicas.

Los espectros infrarrojos se realizaron mediante el método de Reflexión Total Atenuada (ATR) con el fin de que la radiación infrarroja penetrase solamente en la superficie de las partículas. Estos espectros ATR se obtuvieron mediante un equipo Spectrum BX (Perkin-Elmer) en el intervalo 1500-600 $\mathrm{cm}^{-1}$ utilizando una resolución de $1 \mathrm{~cm}^{-1}$. Se realizaron 10 barridos para cada muestra a los cuales se les restó el espectro del fondo (background) en todos los casos. Por otro lado, los espectros Raman se realizaron sobre partículas definidas y observadas con el microscopio óptico a 50 aumentos. Las partículas seleccionadas fueron en cada caso las que correspondían a un tamaño similar (medido de forma automática utilizando el microscopio óptico) al tamaño medio de cada distribución. De esta forma se pretendió que el tamaño de las partículas analizadas fuera análogo entre ambas técnicas, si bien mientras que el equipo ATR se analizan muchas partículas en el equipo Raman se analiza solamente una . El equipo Raman utilizado fue el in Via (Renishaw) iluminado con láser de $514.5 \mathrm{~nm}$ y una potencia de $25 \mathrm{~mW}$. En estas condiciones la penetración del haz láser en la muestra es inferior a $1 \mu \mathrm{m}$, por lo que dicha penetración se puede comparar a la alcanzada con la técnica ATR mencionada anteriormente. Se realizaron 5 espectros Raman para cada muestra. El intervalo analizado fue el de $1500-100 \mathrm{~cm}^{-1}$. Dado que las bandas de las tensiones de deformación de los enlaces Si-O-Si están muy polarizadas, en las medidas Raman se utilizó polarización VV (vertical-vertical) para intensificar dichas bandas. Las áreas de las bandas de los espectros Raman se analizaron mediante el programa de análisis propio del equipo in Via.

\section{RESULTADOS}

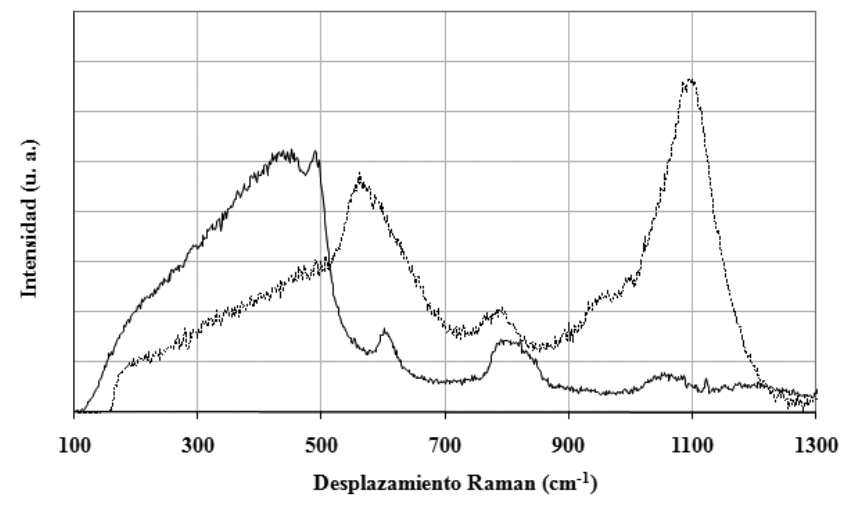

Fig. 1- Espectros Raman de la sílice vítrea (línea continua) y del vidrio (línea discontinua).

\subsection{Sílice vítrea y Vidrio Sin Moler. Espectros Raman y ATR}

Los espectros Raman de la sílice vítrea y del vidrio estudiado están recogidos en la Figura 1. Respecto al espectro correspondiente a la sílice vítrea, en él aparece una banda muy ancha próxima a $440 \mathrm{~cm}^{-1}$ y una banda muy estrecha a $492 \mathrm{~cm}^{-1}$. La banda situada a $440 \mathrm{~cm}^{-1}$ está producida por el movimiento simétrico de las oxígenos puente relativos a los átomos de silicio en una estructura tridimensional $(8,9)$. Por otro lado, la estrecha banda situada a $492 \mathrm{~cm}^{-1}$ se ha atribuido a la vibración de los anillos de cuatro miembros de los tetraédros de $\mathrm{SiO}_{4}$ en la estructura de la sílice, si bien al observar su variación cuando se incorporan diferentes elementos alcalinos, se ha podido asignar al grado de polimerización de la sílice (10). La intensidad relativa de ambas bandas depende de dicho grado de polimerización, como puede verse en los espectros de la Figura 1 así como en la bibliografía. Cuando la sílice vítrea ha sido obtenida en condiciones normales y con alto grado de polimerización, la intensidad de la banda de 440 
$\mathrm{cm}^{-1}$ es superior a la de $492 \mathrm{~cm}^{-1}$, y dicha relación se invierte al disminuir el grado de polimerización.

En estos espectros de la Figura 1 también aparece otra banda de poca intensidad situada a unos $600 \mathrm{~cm}^{-1} \mathrm{y}$ que ha sido atribuida a defectos estructurales en la red de sílice (11, 12). Últimamente dicha banda ha sido asignada a los anillos de $\mathrm{SiO}_{4}$ de tres miembros existentes en la red de sílice. Por otro lado, la banda ancha que aparece sobre los $800 \mathrm{~cm}^{-1}$ está aparentemente asociada con la estructura reticular del vidrio de $\mathrm{SiO}_{2}$ ya que su intensidad disminuye con la despolimerización del vidrio (13). Por último, las bandas situadas a unos 1060 y $1190 \mathrm{~cm}^{-1}$ son probablemente debidas a los modos simétricos y antisimétricos de vibración, respectivamente, de los enlaces Si-O-Si en los que el átomo de oxígeno se mueve paralelo al eje Si-Si.

Además de estas diferentes atribuciones de bandas del espectro Raman de la sílice vítrea, Colomban (14) entre muchos otros investigadores, indicó que en estos espectros se pueden diferenciar dos zonas espectrales cada una correspondiente a diversos modos de vibración. Estas dos zonas corresponden a las de $400-800 \mathrm{~cm}^{-1}$ y $800-1200 \mathrm{~cm}^{1}$, siendo la primera originada por vibraciones de polimerización de la red vítrea, mientras que la segunda es atribuida a grupos estructurales $Q^{n}$, en donde $\mathrm{Q}$ indica el tetraedro $\mathrm{SiO}_{4}$ y $\mathrm{n}$ indica el número de oxígenos no puente existentes.

Respecto al espectro Raman del vidrio analizado, en él aparecen prácticamente las mismas bandas comentadas para la sílice vítrea, si bien sus intensidades están ahora modificadas a la vez que aparece también un nuevo hombro situado sobre los $950 \mathrm{~cm}^{-1}$ que se ha asignado a la rotura de los enlaces Si-O-Si para dar lugar a oxígenos no puente (10, 15). Además de este nuevo hombro, son dos las principales diferencias existentes entre los espectros Raman del vidrio y de la sílice vítrea. Así, para el vidrio, las bandas situadas entre 1000 y $1200 \mathrm{~cm}^{-1}$ son mucho más intensas que las de la

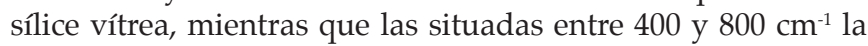
intensidad es muy similar. La otra diferencia está en que en esta segunda zona espectral ahora la banda más intensa está situada sobre los $560 \mathrm{~cm}^{-1}$. Esta nueva banda aparece en la sílice vítrea cuando en ella se ha incorporado una cantidad

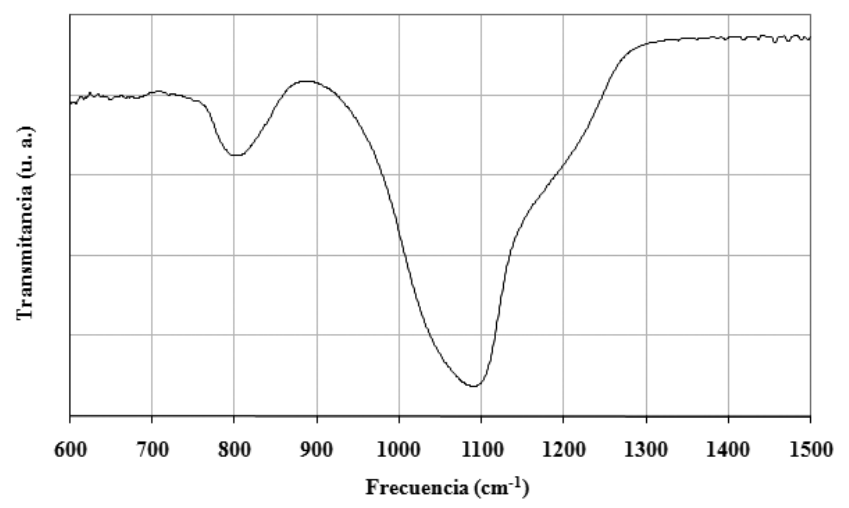

Fig. 2- Espectros IR / ATR de la sílice vítrea (línea continua) y del vidrio (línea discontinua).

superior al 15 en óxidos modificadores de red como $\mathrm{Na}, \mathrm{K}$, Ca, etc. (10).

Los espectros ATR de la sílice vítrea y del vidrio se muestran en la Figura 2. El espectro de la sílice vítrea muestra las dos bandas típicas de los enlaces Si-O-Si situadas a 1100 $\mathrm{cm}^{-1}$ y $800 \mathrm{~cm}^{-1}$, y el hombro situado sobre los $1200 \mathrm{~cm}^{-1}$. La banda y el hombro de mas altas frecuencias de vibración corresponden a las tensiones asimétricas del enlace Si-O-Si en sus componentes ópticas longitudinal (LO) y transversal (TO), respectivamente, mientras que la banda a menor frecuencia corresponde a la tensión simétrica de dicho enlace (16).

Por otro lado, el espectro ATR del vidrio presenta las dos mismas bandas y el hombro, aunque éste está menos definido y desplazado a frecuencias inferiores (estando ahora sobre los $1150 \mathrm{~cm}^{-1}$ ). Así mismo, las dos bandas originadas por las tensiones asimétrica y simétrica están ahora situadas a 985 $\mathrm{cm}^{-1}$ y $770 \mathrm{~cm}^{-1}$, respectivamente. El desplazamiento de las bandas y hombro del vidrio respecto a las de la sílice se debe a la variada composición química de este último mencionada en el Apartado Experimental.

\subsection{Sílice vítrea y Vidrio Molidos. \\ 3.2.1. Espectros Raman}

Los espectros Raman de la sílice vítrea y del vidrio molturados se muestran en las Figuras 3 y 4, respectivamente. Aunque para cada material molturado los espectros son bastante similares, es de destacar que en ambos casos la región espectral comprendida entre 100 y $700 \mathrm{~cm}^{-1}$ disminuye en intensidad con relación a la región espectral comprendida

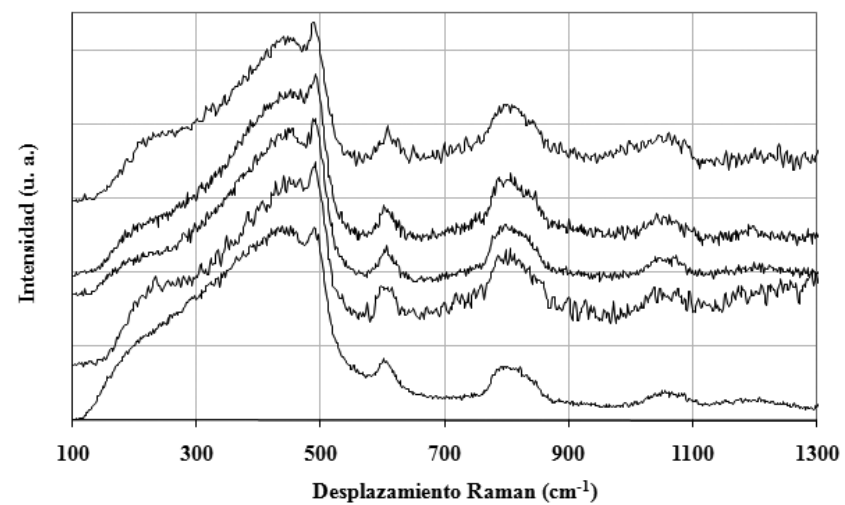

Fig. 3- Espectros Raman de la sílice vítrea molida a diferentes tamaños (valores indicados en micrómetros).

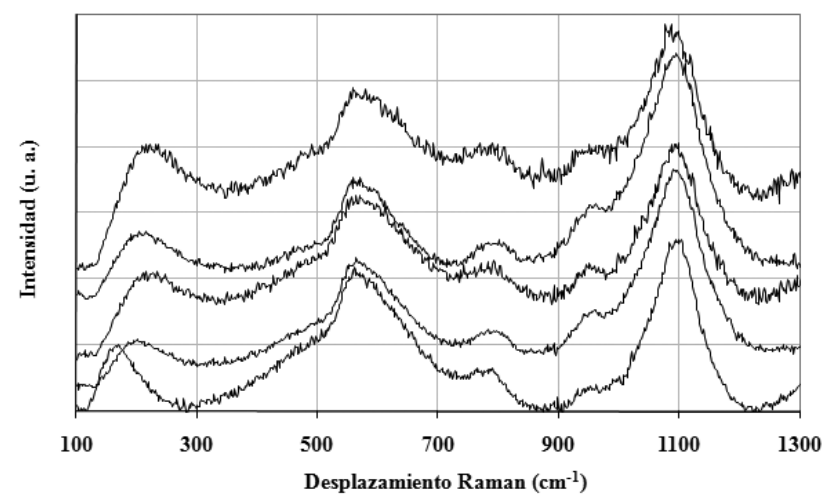

Fig. 4- Espectros Raman del vidrio molido a diferentes tamaños (valores indicados en micrómetros). 
entre 700 y $1300 \mathrm{~cm}^{-1}$. Para el caso concreto de la sílice vítrea, además se aprecia como la banda situada a $492 \mathrm{~cm}^{-1}$ se hace más intensa que la amplia banda situada a $440 \mathrm{~cm}^{-1}$. Se observa también en ambos casos como el ruido de los espectros va aumentando al disminuir el tamaño de partícula, lo que puede ser originado por la dispersión de la radiación y por lo tanto la disminución de la intensidad que llega al detector. En relación con la posición de las bandas de los espectros únicamente se observa una pequeña disminución para la banda situada a $1100 \mathrm{~cm}^{-1}$ en el vidrio, la cual se desplaza hasta $1080 \mathrm{~cm}^{-1}$ al disminuir el tamaño de partícula del vidrio.

\subsubsection{Espectros ATR}

En las figuras 5 y 6 se muestran los espectros IR (ATR) de la sílice vítrea y del vidrio molturados, respectivamente. En estos casos los cambios son más apreciables que los que ocurrían en los espectros Raman. Así, se observa como, al disminuir el tamaño de partícula, las bandas más intensas se desplazan a menores frecuencias a la vez que se van ensanchado. Así, para la sílice vítrea la banda situada a $1080 \mathrm{~cm}^{-1}$ se desplaza a 1080, 1049,1033 y $1031 \mathrm{~cm}^{-1}$ para los tamaños de 100, 50, 30 y 10 $\mu \mathrm{m}$, respectivamente. Ocurre lo mismo con la banda situada a $797 \mathrm{~cm}^{-1}$ si bien en este caso el desplazamiento es menor, siendo ahora las posiciones las de 796, 791, 789 y $788 \mathrm{~cm}^{-1}$, respectivamente para los tamaños indicados. Por otro lado, en el caso del vidrio la banda situada a $983 \mathrm{~cm}^{-1}$ se desplaza a 957, 952, 950 y $951 \mathrm{~cm}^{-1}$ para dichos tamaños de partícula, sin

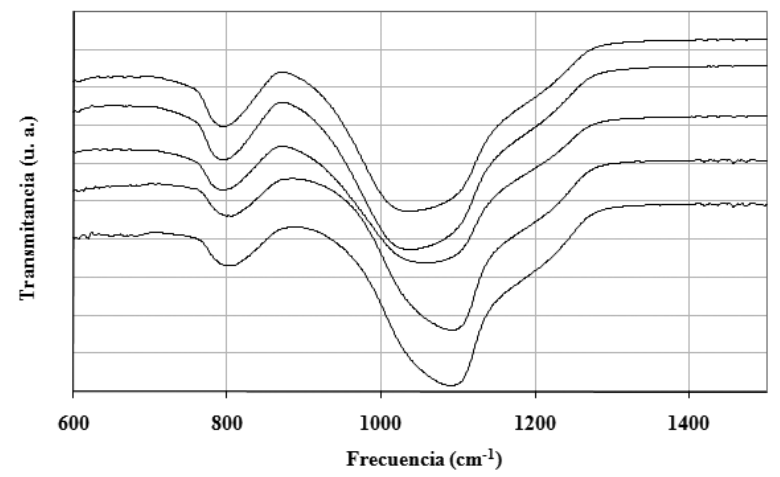

Fig. 5- Espectros IR/ATR de la sílice vítrea molida a diferentes tamaños (valores indicados en micrómetros)

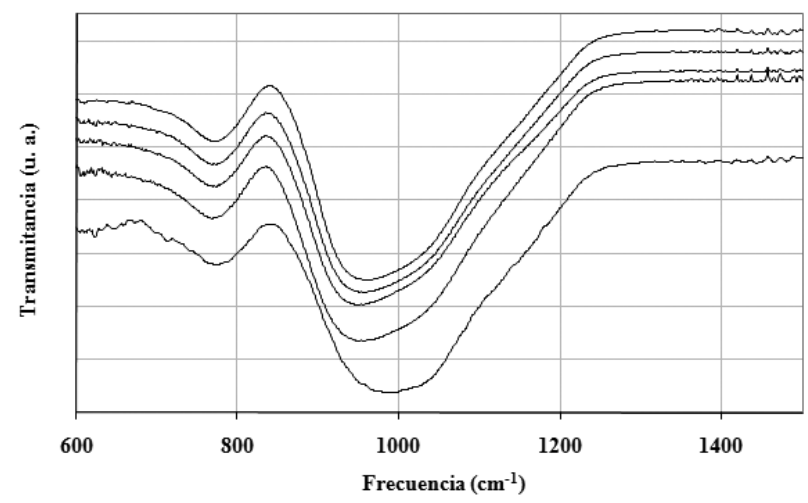

Fig. 6- Espectros IR/ATR del vidrio molido a diferentes tamaños (valores indicados en micrómetros) embargo, la banda situada a $770 \mathrm{~cm}^{-1}$ disminuye a $765 \mathrm{~cm}^{-1} \mathrm{y}$ se mantiene en este valor para todos las tamaños de partícula inferiores a $100 \mu \mathrm{m}$.

\section{DISCUSIÓN.}

Como se ha puesto de manifiesto en las Figuras anteriores, el proceso de molienda tanto de sílice vítrea como de un vidrio convencional modifica los espectros tanto Raman como IR, modificación que se produce tanto en la forma como en la posición de las distintas bandas que aparecen en cada espectro. Este hecho es debido a que durante la molienda la estructura de las partículas se va rompiendo y lo que en principio es estructura vítrea interna se convierte en superficie al ser rota la partícula en otras más pequeñas. Estas roturas inducen amorfizaciones superficiales a corto alcance que pueden ser determinadas bastante bien con medidas espectroscópicas tales como Raman e IR.

Mediante espectroscopia Raman es posible analizar el proceso de amorfización superficial de los materiales vítreos ya que esta técnica permite detectar los diferentes grupos estructurales existentes en los vidrios tal y como han propuesto varios autores $(14,17,18)$. Así, si se hace la deconvolución de los espectros Raman en la región $800-1200 \mathrm{~cm}^{-1}$ aparecen cinco bandas situadas en unos intervalos comprendidos entre $1250-1150 \mathrm{~cm}^{-1}, 1100 \mathrm{~cm}^{-1}, 1100-1050 \mathrm{~cm}^{-1}, 950 \mathrm{~cm}^{-1}$ y 850-800 $\mathrm{cm}^{-1}$, y que corresponden a los grupos o unidades $Q^{4}, Q^{3}, Q^{2}, Q^{1}$ y $Q^{0}$, en donde $\mathrm{Q}$ indica el grupo $\mathrm{SiO}_{4}$ y los superíndices el número de oxígenos puente o unidos a otro átomo de Si. Estos mismos autores indican que en la región 100-800 $\mathrm{cm}^{-1}$ tienen lugar las vibraciones de deformación de estos mismos grupos y que por lo tanto las bandas que aparecen en dicha región pueden ser asignadas a ellos mismos. Esta última afirmación coincide con la expuesta por Matson y col. (10) los cuales comprobaron que la mayor variación que tiene lugar en la sílice vítrea cuando se incorporan elementos alcalinos tiene lugar en la zona de las vibraciones de deformación. Sin embargo, dado que el método de deconvolución puede dar lugar a múltiples soluciones para un mismo espectro y, en el caso de este trabajo, no existe variación de las bandas ya que los materiales solamente se molturan (no se incorpora nada nuevo a ellos), el analizar la evolución de cada banda puede conducir a resultados sin diferencia alguna. En este sentido, Colomban, analizando más de 25 vidrios obtenidos en épocas diferentes, propuso utilizar el índice de polimerización (IP) como una medida adecuada para determinar el grado de despolimerización que tiene lugar en la estructura vítrea cuando es analizada mediante espectroscopia Raman (14). Este IP corresponde a la relación entre las áreas de las dos regiones que aparecen en los espectros Raman, la de las vibraciones de deformación y la de las de tensión ya mencionadas anteriormente. Para que este IP pueda ser utilizado de forma semicuantitativa es necesario que los espectros estén realizados mediante polarización VV, tal y como se indicó en la parte Experimental, ya que de lo contrario las bandas debidas a las vibraciones de deformación son menos intensas al ser bandas que están muy polarizadas en la estructura del vidrio.

La definición de IP puede comprobarse experimentalmente en la Figura 1 en la que la sílice vítrea, la cual debe poseer un grado de polimerización mucho mayor que el del vidrio, posee las bandas más intensas en la región $100-800 \mathrm{~cm}^{-1}$, mientras que el vidrio, el cual está mucho más despolimierizado debido 
a la variada composición química y la cantidad de elementos modificadores, presenta las bandas más intensas en la región $800-1200 \mathrm{~cm}^{-1}$. En la Figura 7 se muestra la variación de IP para la sílice vítrea y para el vidrio estudiados en este trabajo. Se puede comprobar como dicho índice disminuye con el tamaño de partícula o, lo que es lo mismo, con la intensidad de la molienda, lo que indica que la superficie de las partículas está cada vez más despolimerizada al ser molturadas a tamaños cada vez más pequeños. La variación de IP es distinta, sin embargo, para los dos materiales, así para la sílice vítrea el ajuste a una recta da como pendiente $8,6 \times 10^{-3}$, mientras que para el vidrio la pendiente tiene un valor de $8 \times 10^{-4}$, es decir,

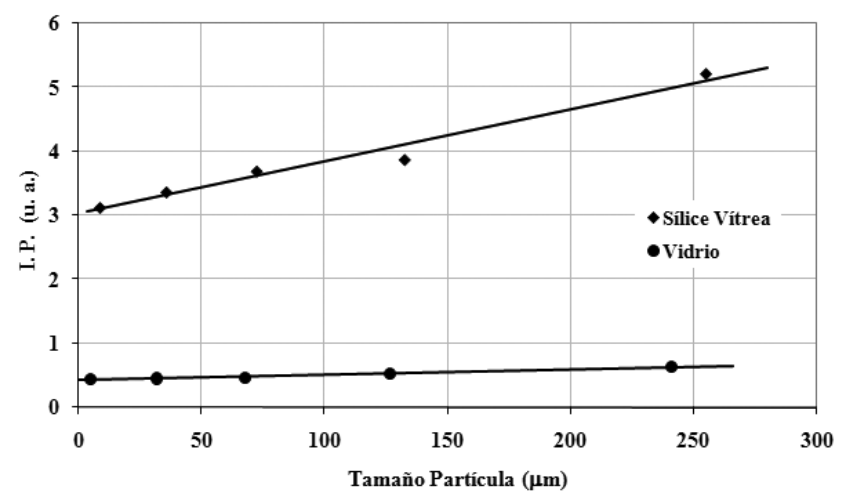

Fig. 7- Variación del índice de polimerización (I. P.) en función del tamaño de partícula.

un valor diez veces inferior. Esto indica que el mayor grado de despolimerización se da en la sílice vítrea, lo que es debido a que por estar más polimerizada que el vidrio, el efecto de la molienda es mayor. Las rectas de ajuste en ambos casos son superiores a 0.97 .

Respecto al análisis de los espectros IR-ATR no existe índice similar al de los espectros Raman lo que es debido a que las bandas están mucho más solapadas y son mucho más anchas. Para el caso del cuarzo si es posible determinar el grado de cristalinidad utilizando el procedimiento establecido por Shoval (19) si bien es necesario que la banda situada a $1145 \mathrm{~cm}^{-1}$ esté bien difenreciada de la situada a $1080 \mathrm{~cm}^{-1}$, hecho que no ocurre en los vidrios ya que hacia $1140 \mathrm{~cm}^{-1}$

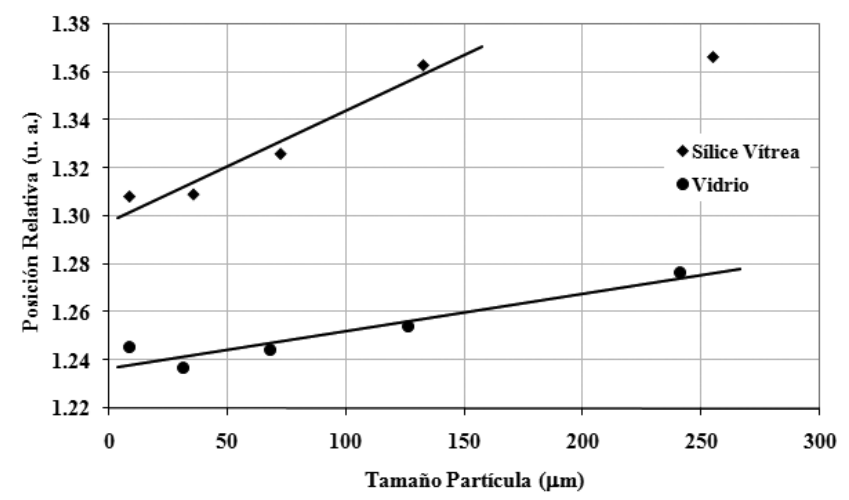

Fig. 8- Variación de la posición relativa de las bandas IR/ATR en función del tamaño de partícula. aparece un hombro solapado con la banda más intensa situada sobre los $990 \mathrm{~cm}^{-1}$. De acuerdo con este hecho y con los resultados comentados sobre la Figura 4, en una primera aproximación, podríamos definir el grado de polimerización como la variación de la posición de las bandas debidas a las vibraciones de tensión asimétrica TO respecto a las de tensión simétrica de los enlaces Si-O-Si ya que, como hemos visto, estas bandas se desplazan a medida que las partículas de la sílice o del vidrio son más pequeñas. En la Figura 8 se muestra dicha variación y puede observarse como en ambos casos se pueden ajustar rectas (excepto para un punto correspondiente a la sílice vítrea) cuyas pendientes son de $5 \times 10^{-4}$ y $2 \times 10^{-4}$, para la sílice vítrea y el vidrio, respectivamente. En este caso la diferencia entre vidrio y sílice vítrea está en 2.5 veces más para ésta última mientras que, como se ha comentado anteriormente, mediante espectroscopia Raman la diferencia era de 10 veces más. Este resultado indica que es la espectroscopia Raman la que permite detectar mejor el grado de polimerización de la estructura vítrea.

\section{CONCLUSIONES.}

Mediante las espectroscopias Raman y ATR/IR se ha seguido la evolución del proceso de molienda tanto de sílice vítrea como de un vidrio convencional. Se ha observado que las bandas son las mismas en cada muestra molida si bien aparecen cambios en las intensidades, en el caso de los espectros Raman, o en las posiciones, en el caso de los espectros IR/ATR. Así, la espectroscopia Raman muestra como las bandas atribuidas a las vibraciones de polimerización de los enlaces Si-O-Si disminuyen al disminuir el tamaño de partícula tanto de la sílice vítrea como del vidrio estudiados. Esta disminución se ha atribuido a un proceso de amorfización o despolimerización superficial causado por la molienda. Por otro lado, la espectroscopia ATR/IR ha mostrado como varían las posiciones relativas entre las bandas atribuidas a las tensiones asimétricas y simétricas de los enlaces Si-O-Si, disminuyendo dicha posición relativa al disminuir el tamaño de partícula de los dos materiales estudiados. Mediante la espectroscopia Raman se ha utilizado el parámetro denominado Índice de Polimerización el cual relaciona las zonas atribuidas a las tensiones de vibración respecto a las de deformación, y se ha observado como dicho índice varía en mayor porcentaje para la sílice vítrea que para el vidrio al disminuir el tamaño de partícula, siendo dicha variación de un orden de magnitud mayor. Sin embargo, mediante la espectroscopia ATR/IR se ha observado que la variación de las posiciones relativas de las bandas al disminuir el tamaño de partícula es solamente tres veces mayor para la sílice vítrea que para el vidrio. Este resultado indica que la espectroscopia Raman es más sensible a los procesos de amorfización superficial que la espctroscopía infrarroja.

\section{AGRADECIMIENTOS}

Este trabajo se ha realizado gracias a la financiación del Ministerio de Educación y Ciencia de España mediante el proyecto CTQ2006-15692-C02-02, y gracias también a la financiación de la Comunidad de Madrid mediante el proyecto S-0505/ PPQ/ 000344. 


\section{BIBLIOGRAFÍA}

1. M. Murat, M. Driuche, Y. Arnaud, A. Bachiorrini, Influence of a long-range grinding on both structural and reactivity changes of quartz, L'industrie Céramique, 847, 3, 197-203 (1990)

2. E. Papirer, J. M. Perrin, B. Siffert, G. Philipponneau, J. M. Laerant The influence of grinding on the surface properties of a-aluminas J. Colloid Interface Sci. 156, 104-108 (1993)

3. H. Yamamura, N. Coda, T. Takayama, K. Ichiba, K, Hamano, Grinding effect of powder on thermal expansion of silica glass powder compacts, J. Ceram. Soc. Japan, Int. Ed. 106, 101-104 (1998)

4. G. Heinicke. Tribochemistry. Ed. Carl Hanser Verlag, Münken, Wien, 1984, p. $287-303$

5. C. Gargori, R. Galindo, J. Badenes, M. A. Tena, G. Monrós, Pigmentos nanoestructurados de base rutilo, Actas del X Congreso Mundial del Azulejo. Qualicer. 2008. Volumen 3. pp. 63-66.

6. A. Kalendová, Effects of particle sizes and shapes of zinc metal on the properties of anticorrosive coatings, Progress in Organic Coatings, 46, 4, 324-332 (2003)

7. E. Papirer, P. Roland, M. Nardin, H. Balard, Variation of the surface energy characteristics of Mica (muscovite) upon grinding, J. Colloid Interface Sci. 113, 1, 62-66 (1986)

8. R. J. Bell, P. Dean, Properties of Vitreous Silica: Analysis of Random Network Models, Nature, 212, 1354-1356 (1966)

9. F. L. Galeener, Band limits and the vibrational spectra of tetrahedral glasses.,Phys. Rev. B 19, 8, 4292-4297 (1979)
10. D. W. Matson, S. K. Sharma, J. A. Philpotts, The structure of high-silica and alkali-silicate glasses. A Raman spectroscopy investigation, J. NonCrystalline Solids, 58, 323-352 (1983)

11. J. B. Bates, R.W. Hendricks, L B. Shaffer, Neutron irradiation effects and structure of non-crystalline SiO2, J. Chem. Phys. 61 4163-4176 (1974)

12. J. C. Mikkelsen, F. L. Galeener, Thermal equilibration of Raman active defects in vitreous silica, J. Non-Crystalline Solids, 37, 71-84 (1980)

13. F. L. Galeener, J. C. Mikkelsen, Jr. Vibrational dynamics in $18 \mathrm{O}-$ substituted vitreous SiO2, Phys. Rev. B23 5527-5530 (1981)

14. P. Colomban, Polymerization degree and Raman identification of ancient glasses used for jewelry, ceramic enamels and mosaics, J. Non-Crystalline Solids 323 180-187 (2003)

15. T. Furukawa, W. B. White, Vibrational spectra and glass structure, J. NonCrystalline Solids, 38/39, 87-92 (1980)

16. M. C. Matos, L. M. Ilharco, R. M. Almeida, The evolution of TEOS to silica gel and glass by vibrational spectroscopy, J. Non-Cristalline Solids, 147/148, 232-236 (1992)

17. D. A. McKeown, I. S. Muller, A. C. Buechele, I. L. Pegg, C. A. Kendzior, Structural characterization of high-zirconia borosilicate glasses using Raman spectroscopy, J. Non-Crystalline Solids, 262, 126-134 (2000)

18. S. Pérez-Villar, J. Rubio, J. L. Oteo, Study of colour and structural changes in silver painted medieval glasses, J. Non-Crystalline Solids, 354, 1833-1844 (2008)

19. S. Shoval, A new method for measuring the crystalline index of quartz by infrared spectroscopy, Miner. Magazine, 55, 579-582 (1991) 